Acta Crystallographica Section E

Structure Reports

Online

ISSN 1600-5368

\section{3,4,5-Trihydroxy- $N^{\prime}-[(1-$ methyl-1H- indol-2-yl)methylidene]benzohydrazide}

\section{Hamid Khaledi, ${ }^{a}$ Siti Munirah Saharin, ${ }^{\text {a }}$ Hapipah Mohd Ali, $^{a *}$ Ward T. Robinson ${ }^{a}$ and Mahmood A. Abdulla ${ }^{b}$}

aDepartment of Chemistry, University of Malaya, 50603 Kuala Lumpur, Malaysia, and ${ }^{\mathbf{b}}$ Department of Molecular Medicine, University of Malaya, 50603 Kuala Lumpur, Malaysia

Correspondence e-mail: hapipah@um.edu.my

Received 30 June 2009; accepted 10 July 2009

Key indicators: single-crystal X-ray study; $T=100 \mathrm{~K}$; mean $\sigma(\mathrm{C}-\mathrm{C})=0.002 \AA$; $R$ factor $=0.044 ; w R$ factor $=0.127 ;$ data-to-parameter ratio $=18.4$.

The structure of the title compound, $\mathrm{C}_{17} \mathrm{H}_{15} \mathrm{~N}_{3} \mathrm{O}_{4}$, displays intermolecular $\mathrm{O}-\mathrm{H} \cdots \mathrm{N}$ and $\mathrm{O}-\mathrm{H} \cdots \mathrm{O}$ hydrogen bonding between adjacent molecules. Intramolecular $\mathrm{O}-\mathrm{H} \cdots \mathrm{O}$ hydrogen bonds also occur. The molecule is essentially planar with a deviation of 0.090 (1) $\AA$ from the best plane running through the connected ring systems.

\section{Related literature}

For related compounds see: Khaledi et al. (2008a,b, 2009).

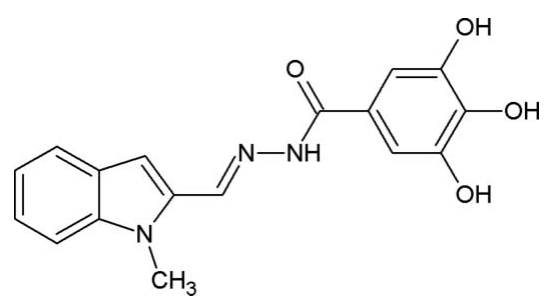

\section{Experimental}

Crystal data

$\mathrm{C}_{17} \mathrm{H}_{15} \mathrm{~N}_{3} \mathrm{O}_{4}$

$M_{r}=325.32$

Monoclinic, $P 2_{1} / n$

$$
\begin{aligned}
& a=9.0839(2) \AA \\
& b=13.1684(3) \AA \\
& c=12.4414(3) \AA
\end{aligned}
$$

$$
\begin{aligned}
& \beta=104.2740(10)^{\circ} \\
& V=1442.30(6) \AA^{3} \\
& Z=4 \\
& \text { Mo } K \alpha \text { radiation }
\end{aligned}
$$$$
\mu=0.11 \mathrm{~mm}^{-1}
$$$$
T=100 \mathrm{~K}
$$$$
0.49 \times 0.16 \times 0.09 \mathrm{~mm}
$$

\section{Data collection}

Bruker APEXII CCD area-detector diffractometer

Absorption correction: multi-scan (SADABS; Sheldrick, 1996)

$T_{\min }=0.948, T_{\max }=0.991$

Refinement

$R\left[F^{2}>2 \sigma\left(F^{2}\right)\right]=0.044$

$w R\left(F^{2}\right)=0.127$

$S=0.99$

4070 reflections

10177 measured reflections 4070 independent reflections 3153 reflections with $I>2 \sigma(I)$ $R_{\text {int }}=0.019$

221 parameters

$\mathrm{H}$-atom parameters constrained

$\Delta \rho_{\max }=0.63{\mathrm{e} \AA^{-3}}^{-3}$

$\Delta \rho_{\min }=-0.26 \mathrm{e}^{-3}$

Table 1

Hydrogen-bond geometry $\left(\AA,{ }^{\circ}\right)$.

\begin{tabular}{lllll}
\hline$D-\mathrm{H} \cdots A$ & $D-\mathrm{H}$ & $\mathrm{H} \cdots A$ & $D \cdots A$ & $D-\mathrm{H} \cdots A$ \\
\hline $\mathrm{O} 2-\mathrm{H} 2 O \cdots \mathrm{O} 4^{\mathrm{i}}$ & 0.84 & 1.80 & $2.6119(14)$ & 164 \\
$\mathrm{O}^{\mathrm{i}}-\mathrm{H} 1 O \cdots \mathrm{N} 2^{\mathrm{i}}$ & 0.84 & 2.06 & $2.7759(15)$ & 142 \\
$\mathrm{O} 3-\mathrm{H} 3 O \cdots \mathrm{O} 2^{\mathrm{ii}}$ & 0.84 & 2.12 & $2.8469(14)$ & 144 \\
$\mathrm{O} 1-\mathrm{H} 1 O \cdots \mathrm{O} 2$ & 0.84 & 2.51 & $2.8570(14)$ & 106 \\
$\mathrm{O} 3-\mathrm{H} 3 O \cdots \mathrm{O} 2$ & 0.84 & 2.31 & $2.7560(14)$ & 113
\end{tabular}

Symmetry codes: (i) $-x+\frac{3}{2}, y-\frac{1}{2},-z+\frac{1}{2}$; (ii) $-x+2,-y,-z$.

Data collection: $A P E X 2$ (Bruker, 2007); cell refinement: $A P E X 2$; data reduction: $S A I N T$ (Bruker, 2007); $\operatorname{program}(\mathrm{s})$ used to solve structure: SHELXS97 (Sheldrick, 2008); program(s) used to refine structure: SHELXL97 (Sheldrick, 2008); molecular graphics: $X$ SEED (Barbour, 2001); software used to prepare material for publication: publCIF (Westrip, 2009).

The authors thank the University of Malaya for funding this study (FRGS grant No. FP009/2008 C).

Supplementary data and figures for this paper are available from the IUCr electronic archives (Reference: HG2532).

\title{
References
}

Barbour, L. J. (2001). J. Supramol. Chem. 1, 189-191.

Bruker (2007). APEX2 and SAINT. Bruker AXS Inc., Madison, Wisconsin, USA..

Khaledi, H., Mohd Ali, H. \& Ng, S. W. (2008a). Acta Cryst. E64, o2108.

Khaledi, H., Mohd Ali, H. \& Ng, S. W. (2008b). Acta Cryst. E64, o2481.

Khaledi, H., Mohd Ali, H. \& Ng, S. W. (2009). Acta Cryst. E65, o169.

Sheldrick, G. M. (1996). SADABS. University of Göttingen, Germany.

Sheldrick, G. M. (2008). Acta Cryst. A64, 112-122.

Westrip, S. P. (2009). publCIF. In preparation. 


\section{supporting information}

Acta Cryst. (2009). E65, o1920 [doi:10.1107/S1600536809027032]

\section{3,4,5-Trihydroxy-N'-[(1-methyl-1H-indol-2-yl)methylidene]benzohydrazide}

\section{Hamid Khaledi, Siti Munirah Saharin, Hapipah Mohd Ali, Ward T. Robinson and Mahmood A. Abdulla}

\section{S1. Experimental}

A mixture of 1-Methylindole-2-carboxaldehyde $(0.80 \mathrm{~g}, 5 \mathrm{mmol})$ and 3,4,5-trihydroxybenzoylhydrazine $(0.92 \mathrm{~g}, 5 \mathrm{mmol})$ in the presence of acetic acid $(1 \mathrm{ml})$ was heated in ethanol $(70 \mathrm{ml})$ for $6 \mathrm{~h}$. The solution was then cooled and filtered to remove the unreacted hydrazine. The filtrate was poured to water $(400 \mathrm{ml})$, the solid product formed were filtered off, washed with diethyl ether,and dried in an oven. Suitable crystals for X-ray analysis were obtained by slow evaporation of an ethanol solution at room temperature.

\section{S2. Refinement}

All Hydrogen atoms were placed at calculated positions ( $\mathrm{C}-\mathrm{H} 0.95-0.98, \mathrm{~N}-\mathrm{H} 0.88$ and $\mathrm{O}-\mathrm{H} 0.84 \AA$ ), with $\mathrm{U}(\mathrm{H})$ set to $1.2-1.5$ times $U_{\text {eq }}(C, N, O)$.

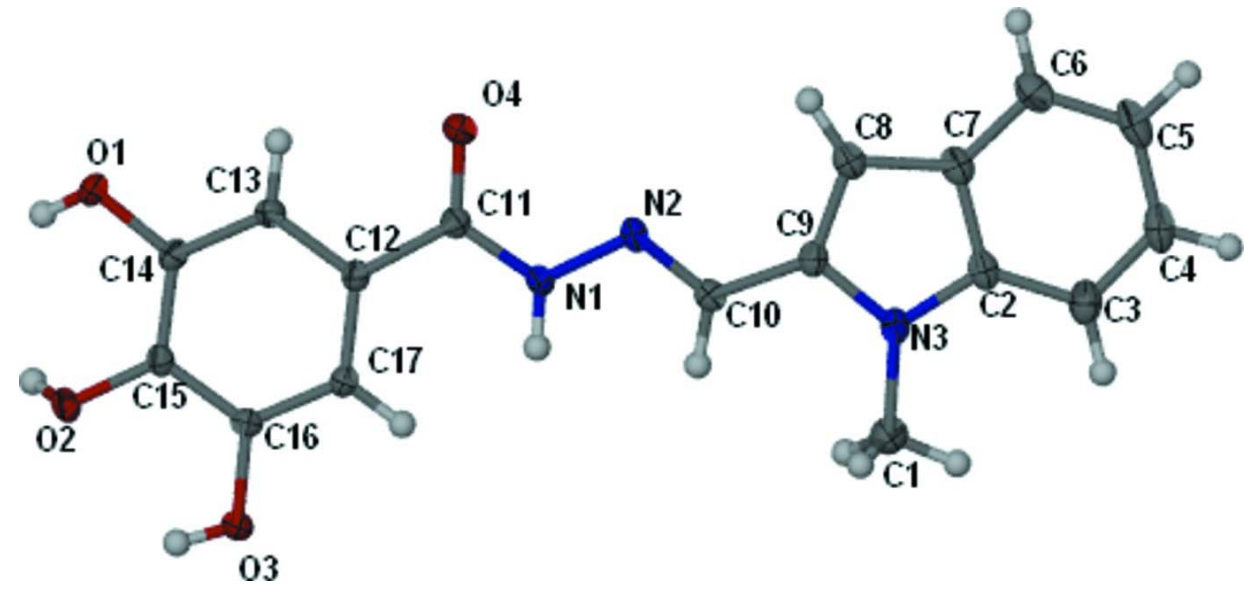

Figure 1

Thermal ellipsoid plot (Barbour, 2001) of the title compound at 70\% probability level. Hydrogen atoms are drawn as spheres of arbitrary radius.

\section{3,4,5-Trihydroxy- $N^{\prime}-[(1-m e t h y l-1 H$-indol-2- yl)methylidene]benzohydrazide}

Crystal data

$\mathrm{C}_{17} \mathrm{H}_{15} \mathrm{~N}_{3} \mathrm{O}_{4}$

$M_{r}=325.32$

Monoclinic, $P 2_{1} / n$

Hall symbol: -P 2 yn

$a=9.0839$ (2) $\AA$

$b=13.1684(3) \AA$

$$
\begin{aligned}
& c=12.4414(3) \AA \\
& \beta=104.274(1)^{\circ} \\
& V=1442.30(6) \AA^{3} \\
& Z=4 \\
& F(000)=680 \\
& D_{\mathrm{x}}=1.498 \mathrm{Mg} \mathrm{m}^{-3}
\end{aligned}
$$


Mo $K \alpha$ radiation, $\lambda=0.71073 \AA$

Cell parameters from 3739 reflections

$\theta=2.3-30.4^{\circ}$

$\mu=0.11 \mathrm{~mm}^{-1}$

Data collection

Bruker APEXII CCD area-detector diffractometer

Radiation source: fine-focus sealed tube

Graphite monochromator

$\omega$ scans

Absorption correction: multi-scan

(SADABS; Sheldrick, 1996)

$T_{\min }=0.948, T_{\max }=0.991$

Refinement

Refinement on $F^{2}$

Least-squares matrix: full

$R\left[F^{2}>2 \sigma\left(F^{2}\right)\right]=0.044$

$w R\left(F^{2}\right)=0.127$

$S=0.99$

4070 reflections

221 parameters

0 restraints

Primary atom site location: structure-invariant direct methods
$T=100 \mathrm{~K}$

Block, green

$0.49 \times 0.16 \times 0.09 \mathrm{~mm}$

10177 measured reflections

4070 independent reflections

3153 reflections with $I>2 \sigma(I)$

$R_{\text {int }}=0.019$

$\theta_{\max }=30.5^{\circ}, \theta_{\min }=2.3^{\circ}$

$h=-9 \rightarrow 12$

$k=-18 \rightarrow 18$

$l=-17 \rightarrow 14$

Secondary atom site location: difference Fourier map

Hydrogen site location: inferred from neighbouring sites

$\mathrm{H}$-atom parameters constrained

$w=1 /\left[\sigma^{2}\left(F_{\mathrm{o}}^{2}\right)+(0.0672 P)^{2}+0.9156 P\right]$

where $P=\left(F_{\mathrm{o}}{ }^{2}+2 F_{\mathrm{c}}{ }^{2}\right) / 3$

$(\Delta / \sigma)_{\max }<0.001$

$\Delta \rho_{\max }=0.63 \mathrm{e} \AA^{-3}$

$\Delta \rho_{\min }=-0.26$ e $\AA^{-3}$

Special details

Geometry. All e.s.d.'s (except the e.s.d. in the dihedral angle between two 1.s. planes) are estimated using the full covariance matrix. The cell e.s.d.'s are taken into account individually in the estimation of e.s.d.'s in distances, angles and torsion angles; correlations between e.s.d.'s in cell parameters are only used when they are defined by crystal symmetry. An approximate (isotropic) treatment of cell e.s.d.'s is used for estimating e.s.d.'s involving 1.s. planes.

Refinement. Refinement of $F^{2}$ against ALL reflections. The weighted $R$-factor $w R$ and goodness of fit $S$ are based on $F^{2}$, conventional $R$-factors $R$ are based on $F$, with $F$ set to zero for negative $F^{2}$. The threshold expression of $F^{2}>\sigma\left(F^{2}\right)$ is used only for calculating $R$-factors(gt) etc. and is not relevant to the choice of reflections for refinement. $R$-factors based on $F^{2}$ are statistically about twice as large as those based on $F$, and $R$ - factors based on ALL data will be even larger.

Fractional atomic coordinates and isotropic or equivalent isotropic displacement parameters $\left(\AA^{2}\right)$

\begin{tabular}{lllll}
\hline & $x$ & $y$ & $z$ & $U_{\text {iso }} * U_{\text {eq }}$ \\
\hline $\mathrm{N} 1$ & $0.47487(13)$ & $0.36671(9)$ & $0.05923(10)$ & $0.0132(2)$ \\
$\mathrm{H} 1 \mathrm{~N}$ & 0.4655 & 0.3333 & -0.0034 & $0.016^{*}$ \\
$\mathrm{~N} 2$ & $0.38051(13)$ & $0.44784(9)$ & $0.06479(10)$ & $0.0134(2)$ \\
$\mathrm{N} 3$ & $0.06173(13)$ & $0.56577(9)$ & $-0.13005(10)$ & $0.0151(2)$ \\
$\mathrm{O} 1$ & $0.99391(12)$ & $0.12533(8)$ & $0.33141(8)$ & $0.0165(2)$ \\
$\mathrm{H} 1 \mathrm{O}$ & 1.0219 & 0.0645 & 0.3313 & $0.025^{*}$ \\
$\mathrm{O} 2$ & $0.98618(11)$ & $0.01716(7)$ & $0.13104(8)$ & $0.0149(2)$ \\
$\mathrm{H} 2 \mathrm{O}$ & 0.9766 & -0.0305 & 0.1737 & $0.022^{*}$ \\
$\mathrm{O} 3$ & $0.77044(12)$ & $0.07206(8)$ & $-0.05744(8)$ & $0.0189(2)$ \\
$\mathrm{H} 3 \mathrm{O}$ & 0.8473 & 0.0349 & -0.0506 & $0.028^{*}$ \\
$\mathrm{O} 4$ & $0.59664(13)$ & $0.38379(8)$ & $0.23996(8)$ & $0.0199(2)$ \\
$\mathrm{C} 1$ & $0.04811(18)$ & $0.51469(13)$ & $-0.23489(13)$ & $0.0226(3)$
\end{tabular}




$\begin{array}{lllll} & & & \\ \text { H1A } & -0.0426 & 0.5393 & -0.2887 & 0.034^{*} \\ \text { H1B } & 0.0393 & 0.4413 & -0.2247 & 0.034^{*} \\ \text { H1C } & 0.1383 & 0.5288 & -0.2623 & 0.034^{*} \\ \text { C2 } & -0.02892(15) & 0.64421(11) & -0.11137(12) & 0.0155(3) \\ \text { C3 } & -0.15129(16) & 0.69271(11) & -0.18421(13) & 0.0192(3) \\ \text { H3 } & -0.1834 & 0.6736 & -0.2600 & 0.023^{*} \\ \text { C4 } & -0.22304(17) & 0.76936(12) & -0.14091(14) & 0.0213(3) \\ \text { H4 } & -0.3059 & 0.8039 & -0.1883 & 0.026^{*} \\ \text { C5 } & -0.17667(17) & 0.79760(11) & -0.02853(14) & 0.0219(3) \\ \text { H5 } & -0.2291 & 0.8505 & -0.0016 & 0.026^{*} \\ \text { C6 } & -0.05636(17) & 0.74988(11) & 0.04323(13) & 0.0195(3) \\ \text { H6 } & -0.0259 & 0.7692 & 0.1191 & 0.023^{*} \\ \text { C7 } & 0.02010(16) & 0.67213(11) & 0.00177(12) & 0.0163(3) \\ \text { C8 } & 0.14341(16) & 0.60759(11) & 0.05098(12) & 0.0162(3) \\ \text { H8 } & 0.1997 & 0.6085 & 0.1263 & 0.019^{*} \\ \text { C9 } & 0.16628(15) & 0.54349(10) & -0.03077(11) & 0.0137(3) \\ \text { C10 } & 0.27384(15) & 0.46201(10) & -0.02389(11) & 0.0134(3) \\ \text { H10 } & 0.2662 & 0.4180 & -0.0856 & 0.016^{*} \\ \text { C11 } & 0.58127(15) & 0.33924(10) & 0.15038(11) & 0.0129(3) \\ \text { C12 } & 0.68091(14) & 0.25165(10) & 0.13972(11) & 0.0123(3) \\ \text { C13 } & 0.78798(15) & 0.22437(10) & 0.23633(11) & 0.0132(3) \\ \text { H13 } & 0.7917 & 0.2597 & 0.3035 & 0.016^{*} \\ \text { C14 } & 0.88907(15) & 0.14590(10) & 0.23472(11) & 0.0128(3) \\ \text { C15 } & 0.88441(15) & 0.09305(10) & 0.13689(11) & 0.0122(2) \\ \text { C16 } & 0.77684(15) & 0.12083(10) & 0.04008(11) & 0.0135(3) \\ \text { C17 } & 0.67475(15) & 0.19925(10) & 0.04105(11) & 0.0143(3) \\ \text { H17 } & 0.6014 & 0.2171 & -0.0249 & 0.017^{*} \\ & & & & \end{array}$

Atomic displacement parameters $\left(\AA^{2}\right)$

\begin{tabular}{lllllll}
\hline & $U^{11}$ & $U^{22}$ & $U^{33}$ & $U^{12}$ & $U^{13}$ & $U^{23}$ \\
\hline $\mathrm{N} 1$ & $0.0140(5)$ & $0.0112(5)$ & $0.0140(5)$ & $0.0024(4)$ & $0.0029(4)$ & $-0.0013(4)$ \\
$\mathrm{N} 2$ & $0.0120(5)$ & $0.0110(5)$ & $0.0173(5)$ & $0.0015(4)$ & $0.0040(4)$ & $0.0006(4)$ \\
$\mathrm{N} 3$ & $0.0132(5)$ & $0.0141(5)$ & $0.0174(6)$ & $0.0019(4)$ & $0.0028(4)$ & $0.0011(4)$ \\
O1 & $0.0180(5)$ & $0.0172(5)$ & $0.0121(4)$ & $0.0047(4)$ & $-0.0006(4)$ & $0.0011(4)$ \\
O2 & $0.0183(5)$ & $0.0122(5)$ & $0.0157(5)$ & $0.0049(4)$ & $0.0069(4)$ & $0.0039(4)$ \\
O3 & $0.0196(5)$ & $0.0214(5)$ & $0.0139(5)$ & $0.0075(4)$ & $0.0010(4)$ & $-0.0046(4)$ \\
O4 & $0.0232(5)$ & $0.0203(5)$ & $0.0151(5)$ & $0.0068(4)$ & $0.0027(4)$ & $-0.0035(4)$ \\
C1 & $0.0219(7)$ & $0.0248(8)$ & $0.0192(7)$ & $0.0035(6)$ & $0.0014(6)$ & $-0.0027(6)$ \\
C2 & $0.0135(6)$ & $0.0124(6)$ & $0.0219(7)$ & $0.0001(5)$ & $0.0066(5)$ & $0.0025(5)$ \\
C3 & $0.0148(6)$ & $0.0182(7)$ & $0.0247(7)$ & $0.0009(5)$ & $0.0050(5)$ & $0.0049(6)$ \\
C4 & $0.0151(6)$ & $0.0168(7)$ & $0.0325(8)$ & $0.0031(5)$ & $0.0070(6)$ & $0.0071(6)$ \\
C5 & $0.0185(7)$ & $0.0134(6)$ & $0.0370(9)$ & $0.0020(5)$ & $0.0128(6)$ & $0.0013(6)$ \\
C6 & $0.0193(7)$ & $0.0154(7)$ & $0.0263(8)$ & $-0.0008(5)$ & $0.0101(6)$ & $-0.0018(5)$ \\
C7 & $0.0149(6)$ & $0.0129(6)$ & $0.0220(7)$ & $-0.0004(5)$ & $0.0068(5)$ & $0.0009(5)$ \\
C8 & $0.0155(6)$ & $0.0142(6)$ & $0.0197(7)$ & $0.0003(5)$ & $0.0061(5)$ & $0.0004(5)$ \\
C9 & $0.0122(6)$ & $0.0123(6)$ & $0.0165(6)$ & $-0.0002(5)$ & $0.0034(5)$ & $0.0022(5)$ \\
C10 & $0.0125(6)$ & $0.0126(6)$ & $0.0155(6)$ & $-0.0008(5)$ & $0.0042(5)$ & $-0.0007(5)$
\end{tabular}


supporting information

\begin{tabular}{lllllll}
$\mathrm{C} 11$ & $0.0134(6)$ & $0.0121(6)$ & $0.0135(6)$ & $0.0001(5)$ & $0.0042(5)$ & $0.0011(5)$ \\
$\mathrm{C} 12$ & $0.0122(6)$ & $0.0116(6)$ & $0.0131(6)$ & $0.0007(4)$ & $0.0031(5)$ & $0.0013(5)$ \\
$\mathrm{C} 13$ & $0.0148(6)$ & $0.0131(6)$ & $0.0120(6)$ & $0.0008(5)$ & $0.0034(5)$ & $-0.0004(5)$ \\
$\mathrm{C} 14$ & $0.0131(6)$ & $0.0132(6)$ & $0.0117(6)$ & $0.0001(5)$ & $0.0022(4)$ & $0.0029(5)$ \\
$\mathrm{C} 15$ & $0.0120(6)$ & $0.0111(6)$ & $0.0142(6)$ & $0.0012(4)$ & $0.0042(5)$ & $0.0020(5)$ \\
$\mathrm{C} 16$ & $0.0147(6)$ & $0.0142(6)$ & $0.0120(6)$ & $0.0006(5)$ & $0.0038(5)$ & $-0.0016(5)$ \\
$\mathrm{C} 17$ & $0.0142(6)$ & $0.0147(6)$ & $0.0129(6)$ & $0.0025(5)$ & $0.0013(5)$ & $0.0007(5)$ \\
\hline
\end{tabular}

Geometric parameters $\left(\AA,{ }^{\circ}\right)$

\begin{tabular}{|c|c|c|c|}
\hline $\mathrm{N} 1-\mathrm{C} 11$ & $1.3455(17)$ & $\mathrm{C} 4-\mathrm{C} 5$ & $1.407(2)$ \\
\hline $\mathrm{N} 1-\mathrm{N} 2$ & $1.3821(15)$ & $\mathrm{C} 4-\mathrm{H} 4$ & 0.9500 \\
\hline $\mathrm{N} 1-\mathrm{H} 1 \mathrm{~N}$ & 0.8800 & $\mathrm{C} 5-\mathrm{C} 6$ & $1.380(2)$ \\
\hline $\mathrm{N} 2-\mathrm{C} 10$ & $1.2900(17)$ & $\mathrm{C} 5-\mathrm{H} 5$ & 0.9500 \\
\hline $\mathrm{N} 3-\mathrm{C} 2$ & $1.3760(18)$ & $\mathrm{C} 6-\mathrm{C} 7$ & $1.405(2)$ \\
\hline N3-C9 & $1.3912(17)$ & C6- 66 & 0.9500 \\
\hline $\mathrm{N} 3-\mathrm{C} 1$ & $1.4455(19)$ & $\mathrm{C} 7-\mathrm{C} 8$ & $1.419(2)$ \\
\hline $\mathrm{O} 1-\mathrm{C} 14$ & $1.3647(16)$ & $\mathrm{C} 8-\mathrm{C} 9$ & $1.376(2)$ \\
\hline $\mathrm{O} 1-\mathrm{H} 1 \mathrm{O}$ & 0.8400 & $\mathrm{C} 8-\mathrm{H} 8$ & 0.9500 \\
\hline $\mathrm{O} 2-\mathrm{C} 15$ & $1.3755(16)$ & $\mathrm{C} 9-\mathrm{C} 10$ & 1.4394 (19) \\
\hline $\mathrm{O} 2-\mathrm{H} 2 \mathrm{O}$ & 0.8400 & $\mathrm{C} 10-\mathrm{H} 10$ & 0.9500 \\
\hline $\mathrm{O} 3-\mathrm{C} 16$ & $1.3613(16)$ & $\mathrm{C} 11-\mathrm{C} 12$ & $1.4921(18)$ \\
\hline $\mathrm{O} 3-\mathrm{H} 3 \mathrm{O}$ & 0.8400 & $\mathrm{C} 12-\mathrm{C} 13$ & $1.3941(18)$ \\
\hline $\mathrm{O} 4-\mathrm{C} 11$ & $1.2365(17)$ & $\mathrm{C} 12-\mathrm{C} 17$ & $1.3972(18)$ \\
\hline $\mathrm{C} 1-\mathrm{H} 1 \mathrm{~A}$ & 0.9800 & $\mathrm{C} 13-\mathrm{C} 14$ & $1.3859(18)$ \\
\hline $\mathrm{C} 1-\mathrm{H} 1 \mathrm{~B}$ & 0.9800 & $\mathrm{C} 13-\mathrm{H} 13$ & 0.9500 \\
\hline $\mathrm{C} 1-\mathrm{H} 1 \mathrm{C}$ & 0.9800 & $\mathrm{C} 14-\mathrm{C} 15$ & $1.3936(18)$ \\
\hline $\mathrm{C} 2-\mathrm{C} 3$ & $1.403(2)$ & $\mathrm{C} 15-\mathrm{C} 16$ & $1.3995(18)$ \\
\hline $\mathrm{C} 2-\mathrm{C} 7$ & $1.416(2)$ & $\mathrm{C} 16-\mathrm{C} 17$ & $1.3900(19)$ \\
\hline $\mathrm{C} 3-\mathrm{C} 4$ & $1.381(2)$ & $\mathrm{C} 17-\mathrm{H} 17$ & 0.9500 \\
\hline $\mathrm{C} 3-\mathrm{H} 3$ & 0.9500 & & \\
\hline $\mathrm{C} 11-\mathrm{N} 1-\mathrm{N} 2$ & $119.41(11)$ & $\mathrm{C} 6-\mathrm{C} 7-\mathrm{C} 8$ & $133.41(14)$ \\
\hline $\mathrm{C} 11-\mathrm{N} 1-\mathrm{H} 1 \mathrm{~N}$ & 120.3 & $\mathrm{C} 2-\mathrm{C} 7-\mathrm{C} 8$ & $107.14(12)$ \\
\hline $\mathrm{N} 2-\mathrm{N} 1-\mathrm{H} 1 \mathrm{~N}$ & 120.3 & $\mathrm{C} 9-\mathrm{C} 8-\mathrm{C} 7$ & $107.18(13)$ \\
\hline $\mathrm{C} 10-\mathrm{N} 2-\mathrm{N} 1$ & $114.41(11)$ & $\mathrm{C} 9-\mathrm{C} 8-\mathrm{H} 8$ & 126.4 \\
\hline $\mathrm{C} 2-\mathrm{N} 3-\mathrm{C} 9$ & $108.32(12)$ & $\mathrm{C} 7-\mathrm{C} 8-\mathrm{H} 8$ & 126.4 \\
\hline $\mathrm{C} 2-\mathrm{N} 3-\mathrm{C} 1$ & $125.46(12)$ & $\mathrm{C} 8-\mathrm{C} 9-\mathrm{N} 3$ & $109.41(12)$ \\
\hline $\mathrm{C} 9-\mathrm{N} 3-\mathrm{C} 1$ & $126.22(12)$ & $\mathrm{C} 8-\mathrm{C} 9-\mathrm{C} 10$ & $129.58(13)$ \\
\hline $\mathrm{C} 14-\mathrm{O} 1-\mathrm{H} 1 \mathrm{O}$ & 109.5 & $\mathrm{~N} 3-\mathrm{C} 9-\mathrm{C} 10$ & $120.98(12)$ \\
\hline $\mathrm{C} 15-\mathrm{O} 2-\mathrm{H} 2 \mathrm{O}$ & 109.5 & $\mathrm{~N} 2-\mathrm{C} 10-\mathrm{C} 9$ & $120.95(13)$ \\
\hline $\mathrm{C} 16-\mathrm{O} 3-\mathrm{H} 3 \mathrm{O}$ & 109.5 & $\mathrm{~N} 2-\mathrm{C} 10-\mathrm{H} 10$ & 119.5 \\
\hline $\mathrm{N} 3-\mathrm{C} 1-\mathrm{H} 1 \mathrm{~A}$ & 109.5 & $\mathrm{C} 9-\mathrm{C} 10-\mathrm{H} 10$ & 119.5 \\
\hline $\mathrm{N} 3-\mathrm{C} 1-\mathrm{H} 1 \mathrm{~B}$ & 109.5 & $\mathrm{O} 4-\mathrm{C} 11-\mathrm{N} 1$ & $122.01(12)$ \\
\hline $\mathrm{H} 1 \mathrm{~A}-\mathrm{C} 1-\mathrm{H} 1 \mathrm{~B}$ & 109.5 & $\mathrm{O} 4-\mathrm{C} 11-\mathrm{C} 12$ & $120.71(12)$ \\
\hline $\mathrm{N} 3-\mathrm{C} 1-\mathrm{H} 1 \mathrm{C}$ & 109.5 & $\mathrm{~N} 1-\mathrm{C} 11-\mathrm{C} 12$ & $117.28(12)$ \\
\hline $\mathrm{H} 1 \mathrm{~A}-\mathrm{C} 1-\mathrm{H} 1 \mathrm{C}$ & 109.5 & $\mathrm{C} 13-\mathrm{C} 12-\mathrm{C} 17$ & $119.91(12)$ \\
\hline $\mathrm{H} 1 \mathrm{~B}-\mathrm{C} 1-\mathrm{H} 1 \mathrm{C}$ & 109.5 & $\mathrm{C} 13-\mathrm{C} 12-\mathrm{C} 11$ & $115.60(12)$ \\
\hline
\end{tabular}




\begin{tabular}{|c|c|c|c|}
\hline $\mathrm{N} 3-\mathrm{C} 2-\mathrm{C} 3$ & $130.13(14)$ & $\mathrm{C} 17-\mathrm{C} 12-\mathrm{C} 11$ & $124.48(12)$ \\
\hline $\mathrm{N} 3-\mathrm{C} 2-\mathrm{C} 7$ & $107.95(12)$ & $\mathrm{C} 14-\mathrm{C} 13-\mathrm{C} 12$ & $120.23(12)$ \\
\hline $\mathrm{C} 3-\mathrm{C} 2-\mathrm{C} 7$ & $121.92(13)$ & $\mathrm{C} 14-\mathrm{C} 13-\mathrm{H} 13$ & 119.9 \\
\hline $\mathrm{C} 4-\mathrm{C} 3-\mathrm{C} 2$ & $117.12(14)$ & $\mathrm{C} 12-\mathrm{C} 13-\mathrm{H} 13$ & 119.9 \\
\hline $\mathrm{C} 4-\mathrm{C} 3-\mathrm{H} 3$ & 121.4 & $\mathrm{O} 1-\mathrm{C} 14-\mathrm{C} 13$ & $117.13(12)$ \\
\hline $\mathrm{C} 2-\mathrm{C} 3-\mathrm{H} 3$ & 121.4 & $\mathrm{O} 1-\mathrm{C} 14-\mathrm{C} 15$ & $122.35(12)$ \\
\hline $\mathrm{C} 3-\mathrm{C} 4-\mathrm{C} 5$ & $121.76(14)$ & $\mathrm{C} 13-\mathrm{C} 14-\mathrm{C} 15$ & $120.49(12)$ \\
\hline $\mathrm{C} 3-\mathrm{C} 4-\mathrm{H} 4$ & 119.1 & $\mathrm{O} 2-\mathrm{C} 15-\mathrm{C} 14$ & $122.20(12)$ \\
\hline $\mathrm{C} 5-\mathrm{C} 4-\mathrm{H} 4$ & 119.1 & $\mathrm{O} 2-\mathrm{C} 15-\mathrm{C} 16$ & $118.70(12)$ \\
\hline $\mathrm{C} 6-\mathrm{C} 5-\mathrm{C} 4$ & $121.17(14)$ & $\mathrm{C} 14-\mathrm{C} 15-\mathrm{C} 16$ & $119.04(12)$ \\
\hline $\mathrm{C} 6-\mathrm{C} 5-\mathrm{H} 5$ & 119.4 & $\mathrm{O} 3-\mathrm{C} 16-\mathrm{C} 17$ & $118.44(12)$ \\
\hline $\mathrm{C} 4-\mathrm{C} 5-\mathrm{H} 5$ & 119.4 & $\mathrm{O} 3-\mathrm{C} 16-\mathrm{C} 15$ & $120.73(12)$ \\
\hline $\mathrm{C} 5-\mathrm{C} 6-\mathrm{C} 7$ & $118.60(15)$ & $\mathrm{C} 17-\mathrm{C} 16-\mathrm{C} 15$ & $120.83(12)$ \\
\hline $\mathrm{C} 5-\mathrm{C} 6-\mathrm{H} 6$ & 120.7 & $\mathrm{C} 16-\mathrm{C} 17-\mathrm{C} 12$ & $119.49(12)$ \\
\hline $\mathrm{C} 7-\mathrm{C} 6-\mathrm{H} 6$ & 120.7 & $\mathrm{C} 16-\mathrm{C} 17-\mathrm{H} 17$ & 120.3 \\
\hline $\mathrm{C} 6-\mathrm{C} 7-\mathrm{C} 2$ & $119.43(13)$ & $\mathrm{C} 12-\mathrm{C} 17-\mathrm{H} 17$ & 120.3 \\
\hline $\mathrm{C} 11-\mathrm{N} 1-\mathrm{N} 2-\mathrm{C} 10$ & $-173.94(12)$ & $\mathrm{C} 8-\mathrm{C} 9-\mathrm{C} 10-\mathrm{N} 2$ & $-9.1(2)$ \\
\hline $\mathrm{C} 9-\mathrm{N} 3-\mathrm{C} 2-\mathrm{C} 3$ & $-179.04(14)$ & $\mathrm{N} 3-\mathrm{C} 9-\mathrm{C} 10-\mathrm{N} 2$ & $172.91(13)$ \\
\hline $\mathrm{C} 1-\mathrm{N} 3-\mathrm{C} 2-\mathrm{C} 3$ & $0.1(2)$ & $\mathrm{N} 2-\mathrm{N} 1-\mathrm{C} 11-\mathrm{O} 4$ & $0.8(2)$ \\
\hline $\mathrm{C} 9-\mathrm{N} 3-\mathrm{C} 2-\mathrm{C} 7$ & $0.29(15)$ & $\mathrm{N} 2-\mathrm{N} 1-\mathrm{C} 11-\mathrm{C} 12$ & $-179.57(11)$ \\
\hline $\mathrm{C} 1-\mathrm{N} 3-\mathrm{C} 2-\mathrm{C} 7$ & $179.39(13)$ & $\mathrm{O} 4-\mathrm{C} 11-\mathrm{C} 12-\mathrm{C} 13$ & $0.73(19)$ \\
\hline $\mathrm{N} 3-\mathrm{C} 2-\mathrm{C} 3-\mathrm{C} 4$ & $179.14(14)$ & $\mathrm{N} 1-\mathrm{C} 11-\mathrm{C} 12-\mathrm{C} 13$ & $-178.95(12)$ \\
\hline $\mathrm{C} 7-\mathrm{C} 2-\mathrm{C} 3-\mathrm{C} 4$ & $-0.1(2)$ & $\mathrm{O} 4-\mathrm{C} 11-\mathrm{C} 12-\mathrm{C} 17$ & $-177.78(13)$ \\
\hline $\mathrm{C} 2-\mathrm{C} 3-\mathrm{C} 4-\mathrm{C} 5$ & $-0.4(2)$ & $\mathrm{N} 1-\mathrm{C} 11-\mathrm{C} 12-\mathrm{C} 17$ & $2.5(2)$ \\
\hline $\mathrm{C} 3-\mathrm{C} 4-\mathrm{C} 5-\mathrm{C} 6$ & $0.3(2)$ & $\mathrm{C} 17-\mathrm{C} 12-\mathrm{C} 13-\mathrm{C} 14$ & $0.5(2)$ \\
\hline $\mathrm{C} 4-\mathrm{C} 5-\mathrm{C} 6-\mathrm{C} 7$ & $0.2(2)$ & $\mathrm{C} 11-\mathrm{C} 12-\mathrm{C} 13-\mathrm{C} 14$ & $-178.13(12)$ \\
\hline $\mathrm{C} 5-\mathrm{C} 6-\mathrm{C} 7-\mathrm{C} 2$ & $-0.7(2)$ & $\mathrm{C} 12-\mathrm{C} 13-\mathrm{C} 14-\mathrm{O} 1$ & $177.99(12)$ \\
\hline $\mathrm{C} 5-\mathrm{C} 6-\mathrm{C} 7-\mathrm{C} 8$ & $-178.83(15)$ & $\mathrm{C} 12-\mathrm{C} 13-\mathrm{C} 14-\mathrm{C} 15$ & $-0.4(2)$ \\
\hline $\mathrm{N} 3-\mathrm{C} 2-\mathrm{C} 7-\mathrm{C} 6$ & $-178.74(13)$ & $\mathrm{O} 1-\mathrm{C} 14-\mathrm{C} 15-\mathrm{O} 2$ & $-0.6(2)$ \\
\hline $\mathrm{C} 3-\mathrm{C} 2-\mathrm{C} 7-\mathrm{C} 6$ & $0.7(2)$ & $\mathrm{C} 13-\mathrm{C} 14-\mathrm{C} 15-\mathrm{O} 2$ & $177.66(12)$ \\
\hline $\mathrm{N} 3-\mathrm{C} 2-\mathrm{C} 7-\mathrm{C} 8$ & $-0.15(16)$ & $\mathrm{O} 1-\mathrm{C} 14-\mathrm{C} 15-\mathrm{C} 16$ & $-177.82(12)$ \\
\hline $\mathrm{C} 3-\mathrm{C} 2-\mathrm{C} 7-\mathrm{C} 8$ & $179.24(13)$ & $\mathrm{C} 13-\mathrm{C} 14-\mathrm{C} 15-\mathrm{C} 16$ & $0.4(2)$ \\
\hline $\mathrm{C} 6-\mathrm{C} 7-\mathrm{C} 8-\mathrm{C} 9$ & $178.27(16)$ & $\mathrm{O} 2-\mathrm{C} 15-\mathrm{C} 16-\mathrm{O} 3$ & $1.6(2)$ \\
\hline $\mathrm{C} 2-\mathrm{C} 7-\mathrm{C} 8-\mathrm{C} 9$ & $-0.04(16)$ & $\mathrm{C} 14-\mathrm{C} 15-\mathrm{C} 16-\mathrm{O} 3$ & $178.93(12)$ \\
\hline $\mathrm{C} 7-\mathrm{C} 8-\mathrm{C} 9-\mathrm{N} 3$ & $0.22(16)$ & $\mathrm{O} 2-\mathrm{C} 15-\mathrm{C} 16-\mathrm{C} 17$ & $-177.94(12)$ \\
\hline $\mathrm{C} 7-\mathrm{C} 8-\mathrm{C} 9-\mathrm{C} 10$ & $-177.98(14)$ & $\mathrm{C} 14-\mathrm{C} 15-\mathrm{C} 16-\mathrm{C} 17$ & $-0.6(2)$ \\
\hline $\mathrm{C} 2-\mathrm{N} 3-\mathrm{C} 9-\mathrm{C} 8$ & $-0.32(16)$ & $\mathrm{O} 3-\mathrm{C} 16-\mathrm{C} 17-\mathrm{C} 12$ & $-178.85(12)$ \\
\hline $\mathrm{C} 1-\mathrm{N} 3-\mathrm{C} 9-\mathrm{C} 8$ & $-179.42(13)$ & $\mathrm{C} 15-\mathrm{C} 16-\mathrm{C} 17-\mathrm{C} 12$ & $0.7(2)$ \\
\hline $\mathrm{C} 2-\mathrm{N} 3-\mathrm{C} 9-\mathrm{C} 10$ & $178.06(12)$ & $\mathrm{C} 13-\mathrm{C} 12-\mathrm{C} 17-\mathrm{C} 16$ & $-0.6(2)$ \\
\hline $\mathrm{C} 1-\mathrm{N} 3-\mathrm{C} 9-\mathrm{C} 10$ & $-1.0(2)$ & $\mathrm{C} 11-\mathrm{C} 12-\mathrm{C} 17-\mathrm{C} 16$ & $177.82(13)$ \\
\hline $\mathrm{N} 1-\mathrm{N} 2-\mathrm{C} 10-\mathrm{C} 9$ & $179.93(12)$ & & \\
\hline
\end{tabular}

Hydrogen-bond geometry $\left(\AA,{ }^{\circ}\right)$

\begin{tabular}{lllll}
\hline$D-\mathrm{H} \cdots A$ & $D-\mathrm{H}$ & $\mathrm{H} \cdots A$ & $D \cdots A$ & $D-\mathrm{H}^{\cdots} \cdots A$ \\
\hline $\mathrm{O} 2-\mathrm{H} 2 O \cdots \mathrm{O} 4^{\mathrm{i}}$ & 0.84 & 1.80 & $2.6119(14)$ & 164 \\
$\mathrm{O} 1-\mathrm{H} 1 O \cdots \mathrm{N} 2^{\mathrm{i}}$ & 0.84 & 2.06 & $2.7759(15)$ & 142
\end{tabular}


supporting information

\begin{tabular}{lllll}
$\mathrm{O} 3-\mathrm{H} 3 O \cdots \mathrm{O} 2^{\mathrm{ii}}$ & 0.84 & 2.12 & $2.8469(14)$ & 144 \\
$\mathrm{O} 1-\mathrm{H} 1 O \cdots \mathrm{O} 2$ & 0.84 & 2.51 & $2.8570(14)$ & 106 \\
$\mathrm{O} 3-\mathrm{H} 3 O \cdots \mathrm{O} 2$ & 0.84 & 2.31 & $2.7560(14)$ & 113 \\
\hline
\end{tabular}

Symmetry codes: (i) $-x+3 / 2, y-1 / 2,-z+1 / 2$; (ii) $-x+2,-y,-z$. 